\title{
Think sepsis to stop deaths, urge advocates
}

\author{
Cite as: CMAJ 2017 September 25;189:E1219-20. doi: 10.1503/cmaj.109-5495
}

Posted on cmajnews.com on Sept. 8, 2017.

$\mathrm{T}$ ory Pereira had never heard of sepsis - a life-threatening response to infection that can lead to organ failure - when she came down with flu-like symptoms and stomach cramps on a work trip to Las Vegas. She was 23 and otherwise healthy, so she expected to tough it out. Then, at a restaurant, her heart stopped.

Paramedics were able to revive Tory and rushed her to hospital. But doctors didn't realize she was septic from a tear in her small intestine until after they discharged her - when she suffered another cardiac arrest.

Tory has joined a growing number of advocates urging clinicians and the public to "think sepsis" before it's too late. "If I had known sepsis was a thing, I would have gone to the hospital 24 hours before I went into cardiac arrest," she says. "Even after I was in septic shock, the doctor still didn't talk about it."

Much later, after emergency surgery, 10 days in a coma and a month in hospital, Tory came across the term "sepsis" in her discharge package. No one at the hospital had used the word, or prepared her for the debilitating fatigue, anxiety and brain fog of post-sepsis syndrome.

"I had six doctors in Las Vegas, but the only thing we were talking about was when I could be discharged," she recounts. "I got another team of doctors when I came home to Georgia and we never talked about it either."

\section{An overlooked killer}

Sepsis is linked to $25 \%-30 \%$ of hospital deaths globally, and up to $50 \%$ in poorer countries and patients with complications, but it's seldom recognized or reported as a leading cause of death and disability. Early diagnosis is crucial and survival rates drop dramatically the longer care is delayed. Yet patients and health care providers often

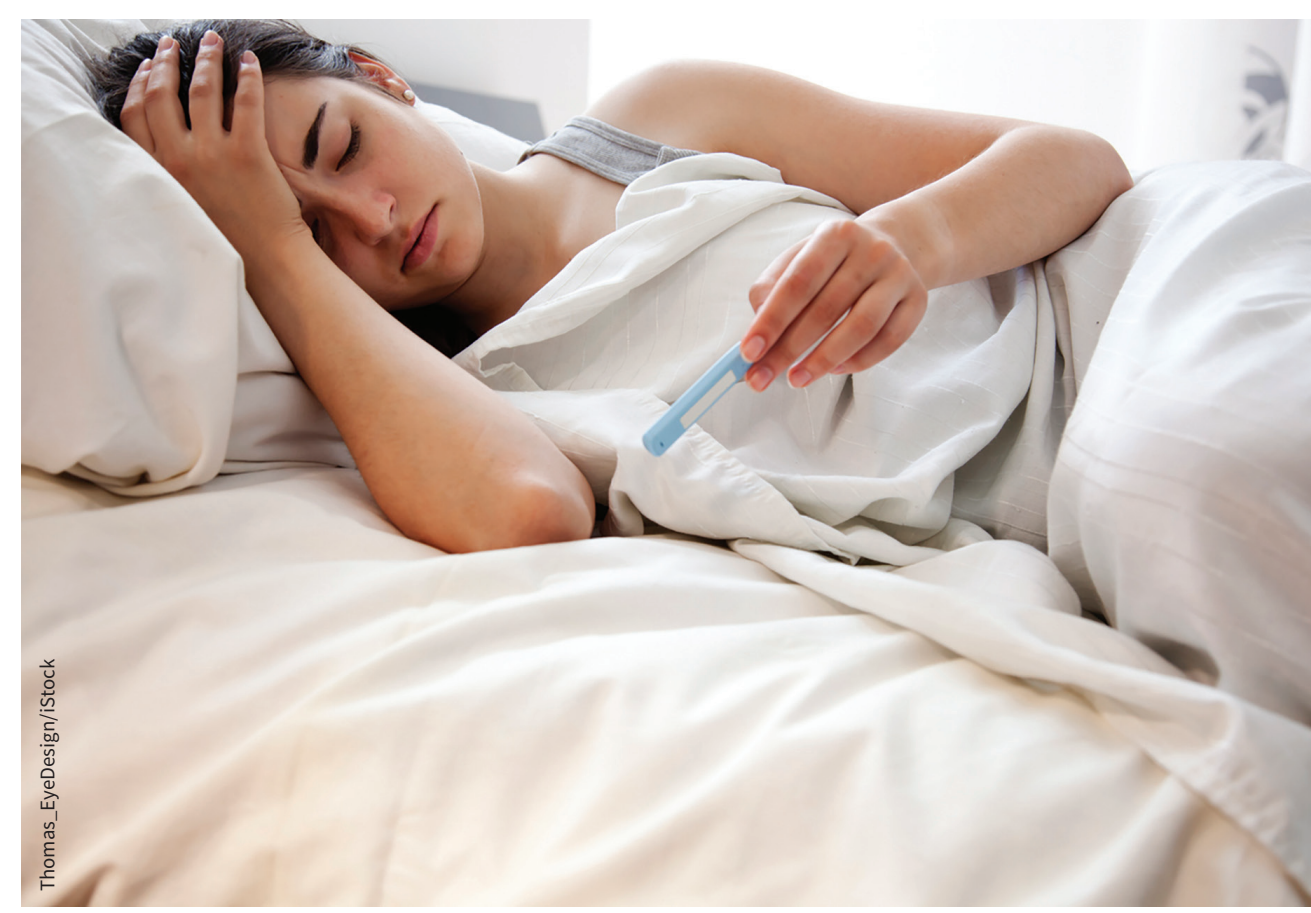

Is it flu or sepsis? Experts say Canadians must start to ask the question more.

miss early signs and symptoms, such as fever and confusion, which may not seem serious at first.

"We've done a very good job campaigning around stroke, telling people to think fast if they are experiencing blurry vision or weakness," says Dr. Sagar Dugani, a Toronto-based internist. "We have to have a similar campaign educating people to think about sepsis."

"The problem with sepsis is that it could look like a lot of other things," Dugani explains. Even clinicians "don't use the word sepsis often," further complicating matters. Because sepsis can result from a number of infections and conditions, people tend to focus on the underlying cause, he explains. "A lot of people will say this is pneumonia, or this is a bad urinary tract infection."

This also makes it difficult to estimate the true burden of sepsis, because patients may not be counted unless doctors indicate sepsis as a diagnosis on their charts. "I get patients coming through my door where everybody's skirted around that," says Dr. Alison Fox-Robichaud, a critical care specialist at Hamilton Health Sciences in Ontario and chair of the Canadian Sepsis Foundation. "They've written tachycardia and fever and confusion, but they haven't written sepsis."

Poor awareness has contributed to dangerous delays with heavy costs for both patients and health systems, says Dr. Niranjan Kissoon, vice-president of medical affairs for BC Children's Hospital and vice-chair of the Global Sepsis Alliance. He cited a 2015 review by the Canadian Medical Protective Association that showed "inadequate patient assessment" was a factor in $75 \%$ of 327 medical lawsuits and complaints involving sepsis. 
"We don't recognize it very well and we don't treat it as quickly as possible," Kissoon says. In addition to saving lives, "giving antibiotics earlier reduces the need for intensive care and expensive care, so you save a lot by treating earlier."

Sepsis cost the United States' Medicare system more than US\$6 billion in 2015, according to recent reports. And this may not factor in the additional costs associated with readmissions of patients who have had sepsis, including for symptoms of post-sepsis syndrome. "Many of these patients, $30 \%$ or $40 \%$, will have problems with memory loss, pain, appetite and post-traumatic stress," explains Kissoon. "They cannot function, so sepsis is a tremendous economic burden, too."

\section{New momentum}

Tragic cases have brought a new spotlight to the issue in recent years. The death of a 12-year-old spurred New York State to require all hospitals to adopt protocols for early diagnosis and treatment of sepsis, reportedly reducing adult patients' odds of dying by $21 \%$. Other states have since introduced similar protocols.

The case was a "watershed moment" for Americans, says Dr. Christopher Seymour, an assistant professor of critical care and emergency medicine at the University of Pittsburgh. "It stimulated much of the effort at the US Centers for Disease Control as well, to track and survey sepsis in a way that's never been done before," he says.

Kissoon notes that there have been "seismic gains" in global awareness in the past year, including a resolution by the World Health Assembly recognizing sepsis as a major threat. The resolution dedicates funds to combat the condition and requires the World Health Organization to report back on progress in three years.

But as political leaders in Germany and the United Kingdom have championed action on sepsis, "Canada has lagged behind," Kissoon says. He was involved in launching the Canadian Sepsis Foundation to shine a national spotlight on the issue.

"You've got to fight the disease in the corridors of authority and power to get the money to fight it at the bedside," he says. "It's really getting champions at the political level that we need right now."

Fox-Robichaud argues that Canadian federal and provincial governments could start by improving how they track sepsis. "We need a national plan for data gathering" that would clarify, among other things, how sepsis is defined and coded, she says.

Seymour agrees the first step is to "get everyone to speak the same language," followed by establishing registries of sepsis cases and deaths. "It all starts with knowing how common and deadly your syndrome is, and that's not only to help educate clinicians in caring for the patient, but also the funders who would support innovative research."

Governments also have an upstream role to play in preventing infections, says Kissoon. This includes promoting hand hygiene, the use of safe-surgery checklists, breastfeeding to prevent infections in the first six months of life, and vaccination, as "many cases of sepsis are from vaccine-preventable diseases."

"The world is becoming more and more set up for sepsis," Kissoon warns, citing the increasing risks of infectious disease outbreaks from urbanization, mass migration and climate change. Reducing sepsis deaths will require tackling these social and environmental challenges, alongside the growing threat of antibiotic resistance, he says.

"Everyone has to realize if we continue in this trend, if we don't have antimicrobial agents, we are going to set back medicine almost 50 years where you cannot do surgery and other things without risk of major infections."

\section{Lauren Vogel, CMAJ}

Editor's note: Drs. Dugani and Kissoon are members of the CMAJ Editorial Advisory Board and were not involved in the editorial decision-making process for this article. 